\section{Sentidos de lo}

común en la educación

comunitaria: a propósito

de la Universidad

Pedagógica Nacional
Segundo semestre de $2020 \cdot$ pp. 205-219

Segunda época

N. ${ }^{0}$
Meanings of the

Common in the

Community Education:

About Universidad

Pedagógica Nacional
Sentidos do comum

na educação

comunitária: sobre

a Universidad

Pedagógica Nacional

Amadeo Clavijo Ramírez* https://orcid.org/0000-0001-9015-5310

Alcira Aguilera Morales**

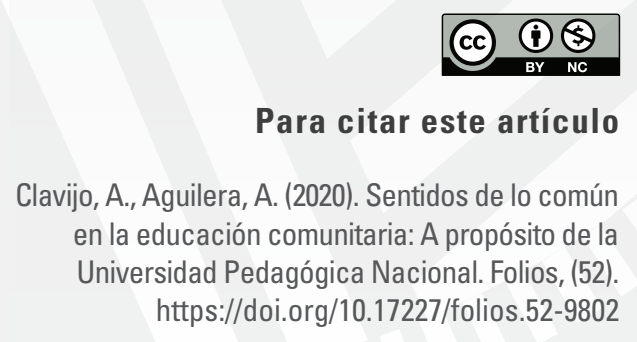

Para citar este artículo

jo, A., Aguilera, A. (2020). Sentidos de lo común en la educación comunitaria: A propósito de la https://doi.org/10.17227/folios.52-9802

* Profesor investigador de la Universidad Pedagógica Nacional, Bogotá, Colombia. Miembro del grupo de investigación Polifonías de la Educación Comunitaria y Popular. Correo electrónico: aclavijo@pedagogica.edu.co.

* Profesora investigadora de la Universidad Pedagógica Nacional, Bogotá, Colombia. Miembro del grupo de investigación Sujetos y Nuevas Narrativas en la Investigación y la Enseñanza de las Ciencias Sociales, de la Universidad Pedagógica Nacional. Correo electrónico: aamorales@pedagogica.edu.co. 


\title{
Resumen
}

El artículo es producto del proyecto de investigación “Construcción de la memoria colectiva de tres propuestas de formación en educación comunitaria de la UPN". El objetivo del proyecto fue recuperar la memoria de la educación comunitaria en la Universidad Pedagógica Nacional de Colombia (UPN). En esta tarea se acudió a la reconstrucción de la trayectoria histórica de tres experiencias de educación comunitaria adelantadas en la UPN desde la década de los setenta hasta finales del siglo xx. Así, a partir de la recuperación y el análisis de las fuentes orales y documentales se aportó a la conceptualización de una de las tradiciones pedagógicas de la universidad. En este rastreo histórico indagamos por la relación entre los sentidos de lo común y la educación comunitaria a partir de tres problematizaciones: sentidos formativos en términos ético-políticos; lo común como insurgente epistémico; y lo solidario en la educación comunitaria. Ellos no solo permiten la visibilización de estas experiencias, sino que además nos llevan a nuevas claves para leerlas con la intención de proyectar discusiones que se adelantan actualmente en el campo de la educación comunitaria en el ámbito universitario.

\section{Palabras clave}

educación comunitaria; común; sentidos formativos

\begin{abstract}
This article is a product of the research project "Construction of the Collective Memory of Three Proposals for Training in Community Education of the UPN". The objective of the project was to recover the memory of community education at the Universidad Pedagógica Nacional de Colombia (UPN). In this task, the authors reconstruct the historical trajectory of three community education experiences developed at the UPN through the seventies to the late 1990s. Thus, from the recovery and analysis of oral and documentary sources, the study contributes to the conceptualization of one of the university's pedagogical traditions. In this historical tracking, we inquire about the relationship between the meanings of the common and community education, based on three problematizations: formative senses in ethical-political terms; the common as an epistemic insurgent; and solidarity in community education. They allow the visibility of these experiences, but also provide new keys to read them to project current discussions on the field of community education taking place at university.
\end{abstract}

\section{Keywords}

community education; common; formative senses

\section{Resumo}

0 artigo é produto do projeto de pesquisa “Construção da memória coletiva de três propostas de treinamento em educação comunitária na UPN" 0 objetivo do projeto foi recuperar a memória da educação comunitária na Universidade Pedagógica Nacional da Colômbia (UPN). Nesta tarefa, foi realizada a reconstrução da trajetória histórica de três experiências de educação comunitária realizadas no início da década de 1970 até o final do século Xx. Assim, a partir da recuperação e análise das fontes orais e documentais, contribuiu para a conceituação de uma das tradições pedagógicas da universidade. Nesta pesquisa histórica, investigamos a relação entre os sentidos da educação comum e da comunidade com base em três problematizações: sentidos formativos em termos ético-políticos; o comum como insurgente epistêmico; e solidariedade na educação da comunidade. Eles não apenas permitem a visibilidade dessas experiências, mas também nos levam a novas chaves para lê-las com a intenção de projetar discussões que estão ocorrendo atualmente no campo da educação comunitária no ambiente universitário.

\section{Palavras chave}

Comunidade; educação comum; sentidos formativos 
Son ya cuarenta y siete años de historia de la educación comunitaria (ECO) en la Universidad Pedagógica Nacional de Colombia. Este recorrido se inicia a comienzos de los años setenta con el Centro de Educación de Adultos CEA, escenario de las primeras construcciones epistémicas de lo que hoy se llaman formalmente sentidos de lo comunitario (Torres, 1997). El CEA mantuvo labores formativas de 1973 a 1979, periodo en el cual generó posibilidades educativas, principalmente, para los trabajadores de la universidad. Después, podemos mencionar el Programa de Educación Comunitaria, recordado como Preco, que se adelantó entre los años 1984 y 1993, con el cual diferentes programas de pregrado de la Universidad tuvieron lugar en las comunidades del suroriente de Bogotá a través de las prácticas comunitarias, desde las cuales se buscaba incidir en el contexto urbano popular y en la propia formación de los futuros docentes. Finalmente, el Programa Educativo para la Paz y la Reconciliación Nacional (PEPRN), que entre 1991 y 1998 contribuyó a la formación de excombatientes provenientes de los grupos guerrilleros desmovilizados a inicios de los noventa, y contó con procesos y programas asociados a la educación comunitaria. Desde la revisión de estas trayectorias históricas y la memoria colectiva de las tres experiencias en mención arriesgamos una lectura de qué es lo común en la educación comunitaria. ${ }^{1}$

La formalización del debate de la ECO en la UPN permitió acceder a otras fuentes que desde contextos diversos problematizaron las perspectivas de lo que se nominaba educación comunitaria. Así, Gadotti (1993) planteaba el problema del posicionamiento político y las implicaciones para la formación del sujeto que tenía la educación comunitaria. Quintana (1991) reposicionaba la categoría Educación comunitaria de adultos y abría el debate desde el concepto de educación permanente, entendida como Eco Sistema Educativo; cuestionaba el papel hegemónico

1 La investigación se ubica en una convocatoria específica que busca recuperar el patrimonio pedagógico, los saberes pedagógicos y didácticos de la Universidad Pedagógica Nacional de Colombia. En ella participaron además los profesores Alfonso Torres, Víctor Manuel Rodríguez e Isabel González Terreros. del discurso del desarrollo comunitario para decantar su postura en la categoría Acción territorial de educación permanente. De esta manera se buscaba desligar la idea de una educación comunitaria de carácter funcionalista para explorar sus perspectivas emancipatorias.

Estableciendo las relaciones entre comunidad, rasgos de lo comunitario y educación comunitaria, el profesor Alfonso Torres (1997) planteaba que las posibilidades de la educación comunitaria estarían en su capacidad de articularse y potenciar dichos modos de la vida social, política, y cultural de las comunidades. Sin embargo, el autor nos advertía que si la práctica educativa privilegiaba exclusivamente la dimensión comunitaria de afirmación grupal, podría caer en el tan cuestionado comunitarismo. En esta misma perspectiva, Sierra (2000) propuso las relaciones entre ECO y Pedagogía popular enfatizando en los aportes de la educación popular (EP) al campo pedagógico de la ECO, en sentido metodológico de participación y empoderamiento. Recabó además en la crítica hacia quienes entendieron la ECO como emergente del proceso de refundamentación de la educación popular. En esta misma discusión, Clavijo (2002) cuestionó la autonomía e independencia de la ECO con relación a la educación popular y planteó el interrogante sobre si la ECO realmente era un campo independiente y autónomo. Recogiendo su tesis, Clavijo (2016) ha sostenido la necesidad de abordar la construcción del campo de la ECO, a partir de sus tensiones políticas y finalidades educativas, entendiendo que esta debe superar el concepto de "campo emergente" para posicionar una trayectoria práxica, la cual ha consolidado en los últimos veinte años (Clavijo, 2017).

Todos estos constructos han fortalecido el campo conceptual de la ECo. Por esta razón, recuperar la tradición educativa comunitaria, localizarla y visibilizarla desde los aportes que se han hecho desde la Universidad Pedagógica Nacional de Colombia implica recuperar las construcciones en la década de los setenta hasta mediados de los ochenta como antecedentes fundacionales para su posterior formalización hacia la mitad de la década de los noventa. 
No se puede desconocer que la articulación con otras experiencias ha coadyuvado a posicionar esta discusión en el ámbito de la academia.

En atención a esta discusión, que por demás se relaciona temporalmente con las experiencias documentadas, centramos ahora nuestro propósito en las interdependencias entre lo común y la educación comunitaria que configuran la ruta analítica del texto aquí presentado, reiteramos, desde la recuperación histórica de esta tradición dentro de la universidad.

\section{Los sentidos formativos: el tránsito del individuo al sujeto}

En el contexto de las experiencias de las que estamos hablando, no es posible concebir la formación sin sentido ético-político, pues más allá de que se entienda como "el cultivo de los talentos para el perfeccionamiento propio [o] [...] bildung" (Fabre, citado en Aguilera, González y Torres, 2015, p. 16), es la formación un ejercicio reflexivo que permite visualizar y valorar, desde la experiencia, el camino que se está transitando con la perspectiva de generar empoderamientos en torno a los procesos propios que permitan potenciar prácticas transformativas en los sujetos con relación a sus cotidianidades. Tales sentidos han estado claros en los propósitos e intencionalidades de los programas educativos en cuestión; para el CEA es "[...] la experiencia directa de una comunidad de diálogo educativo, identificada en un único propósito: Aportar al desarrollo integral del adulto como sujeto activo, transformador de su medio social" (Sequeda 1980, p. 10).

Las búsquedas en la formación del sujeto en el CEA llevaron a establecer perspectivas curriculares, en las que la elección de contenidos, metodologías y demás aspectos permitiera a los sujetos en formación lecturas críticas de realidad, desde las cuales no solo se cuestiona sino que se rediseñan transformaciones de modos de existencia. En palabras del profesor Mario Sequeda,

Esta concepción alternativa del quehacer educativo, supone un esfuerzo permanente por superar la enseñanza magistral, para lo cual se requiere una actitud de búsqueda permanente e invención de nuevos métodos y técnicas, que a partir de los intereses, necesidades y problemas de la vida del adulto provea condiciones para que apropie y desarrolle un saber crítico. Teniendo en cuenta que la realidad a que se enfrenta el individuo es de carácter problemático, su acercamiento a la misma, su modo de conocerla y transformarla debe implicar el manejo de un conjunto de instrumentos y de conocimientos de diversas disciplinas que le permitan interpretar su complejidad y plantear alternativas de solución. (1979, p. 12).

Consecuente con tal postura política, Sequeda (1977) propone para el CEA la ruta ética, que en nuestro lenguaje la apropiamos en horizontes de sentido: "Bienes sociales, fraternidad e igualdad y supresión de toda forma de dominación y la solidaridad en un sentido común" (p. 1). Esta perspectiva de formación y constitución del sujeto se puede rastrear en la experiencia educativa del Preco, donde se identifica un compromiso con la transformación del sujeto, así,

[...] el educador comunitario avanza por exigencia de su grupo en un proceso cualitativo de transformación que podría sintetizarse así:

Tránsito de un quehacer ocasional o coyuntural a un quehacer orgánico.

Tránsito de un quehacer empírico a un quehacer fundamentado en la articulación teoría- práctica.

Tránsito de una apropiación individual del saber a una apropiación colectiva del mismo.

Tránsito de una reproducción ingenua de modelos formativos a la construcción de una pedagogía popular (Sequeda et al. 1985, p. 8).

Los tránsitos enunciados, que bien podríamos apropiar como sentidos comunes de la formación, se instituyen entonces, en ese caminar del individuo al sujeto social, quien se hace consciente de los procesos de sujeción y subjetivación que condicionan su convivencia en comunidad. En ese orden de ideas, la formación de sujetos adquiere sentidos políticos 
desde la constitución de pensamiento crítico y sentidos éticos a partir de la responsabilidad de asumir el bien común y la transformación social como formas de vida.

Esta tarea es mediada por propuestas formativas en las que se construyen aspectos curriculares acompañados de prácticas reflexivas y con la voz de todos los actores del proceso, pues es de esta manera que se hace evidente el tránsito del individuo al sujeto. Así se generan afecciones en el otro, como una manera de posibilitar sus desplazamientos y, por qué no, su transformación:

Se trata de reconstruir un proceso con el cual se está comprometido. Se intenta desde dentro del proceso histórico-social, condensar algunos elementos teórico-prácticos que nos inciten a seguir la marcha, acompañando los sectores populares en su lucha de cada día por leer la realidad para escribir la historia con rasgos nuevos [...] no puede haber acción transformadora de la realidad social y cultural sin que medie un proceso de reflexión sobre la misma práctica y simultáneamente de impulso a procesos de organización y movilización social. (Sequeda et al. 1985, pp. 4-5).

Unos años más tarde, desde la propuesta curricular de la práctica pedagógica para estudiantes de Licenciatura en Ciencias Sociales, el profesor Alfonso Torres se preguntaría por las metodologías que permitieran construir un enfoque investigativo y pedagógico que, en primer lugar, se ocupara de la lectura de contexto por parte de los educadores en formación y, en segundo lugar, de la participación de los pobladores en el proceso investigativo:

La elaboración de un programa de sociales que "parta de la realidad" de los estudiantes, demanda con urgencia estudios sistemáticos sobre la historia de tales prácticas individuales y colectivas de los barrios a los que pertenecen. Sin embargo, su reconstrucción desde la práctica Docente debe ser "educativa" y no simplemente "investigativa". Por ello se ha determinado como estrategia metodológica su carácter participativo y organizativo, es decir, no debe ser tarea exclusiva y aislada de los practicantes sino trabajo colectivo con los grupos de base y con otras personas del barrio interesadas en el trabajo histórico. Logramos constituir un equipo de 8 personas voluntarias [...] con ellas, una vez elaborado un plan tentativo, hicieron una primera exploración bibliográfica sobre la temática barrial y sobre el barrio en particular. (Torres, 1989, pp. 21-22).

Involucrar a la comunidad en el proceso de construcción educativa e investigativa ha sido una propuesta particular de los proyectos educativos alternativos, pues es con la comunidad que el currículo se fundamenta en la realidad porque trata de problemas que atañen al contexto. En ello coinciden Sequeda y Torres, quienes comparten sus miradas respecto al papel de la experiencia en la constitución del sujeto.

En la misma línea, a comienzos de la década de los noventa, el PEPRN, en su proyecto educativo y pedagógico, planteaba:

El proceso pedagógico está orientado a gestar espacios que permitan que el conocimiento y la experiencia educativos lleven al participante a la comprensión de la realidad local, regional y nacional; favorezcan un descentramiento o toma de distancia frente a la realidad inmediata para contextualizar, racionalizar, resignificar y proyectar en términos individuales y colectivos una posibilidad cierta de futuro. (Gallo, 1994, p. 4).

El descentramiento del individuo ante su realidad produce incomodidad, pues es un ejercicio de deslocalización, que se constituye en un primer paso para la constitución de sujeto, luego se produce un segundo paso que implica contextualizar y resignificar para comprender y en esa perspectiva poder proyectar. Para Vladimir Zabala, uno de los pioneros del PEPRN significa:

Todo ese ir y venir es un trabajo de pensamiento y de construcción comunitaria que se traduce en los 4 mínimos: la sintonía de lo mínimo necesario, el acuerdo mínimo necesario, el esfuerzo crítico mínimo, y la certeza mínima de lo útil, esto se va construyendo invisiblemente en la sociedad (en el proceso se dio eso habían [sic] sintonías y desacuerdos, peleas durísimas), superado esto, luego se pasa al nivel de la cedula [sic] de campo: autoconciencia de la común unidad, autosostenimiento 
del proyecto, de la autogestión y de la autonomía. Luego a nivel de la ficha de campo, se da la organización, la confianza mutua, ayuda mutua, respeto mutuo y el acuerdo mutuo, sin esto, no se puede dar lo organizativo. Luego se llega al máximo poder comunitario, que se da en la matriz de campo, es el máximo logro posible, la máxima transformación, el máximo cambio posible, y la máxima potencia comunitaria. Lo que más pueda la sociedad para cambiar su entorno, esas son las acciones comunitarias. (Zavala, entrevista. En Clavijo, 2002, p. 65).

El proyecto educativo pretendía llevar al individuo en un proceso formativo pasando por las condiciones mínimas necesarias a nivel individual, hasta llegar al desarrollo máximo posible, que se reflejaría en las capacidades transformadoras del poder comunitario. Lo anterior coincide con el esquema metodológico propuesto por Zemelman (1992), en el proceso de constitución de sujetos sociales el cual: "[...] se fundamenta en la idea de niveles de realidad que están articulados entre sí: [...] I. Individual. II. Colectivo. III. Utopía. IV. Proyecto. v. Fuerza" (17). Estos niveles de acuerdo con el autor no son lineales, pues se pueden experienciar en simultaneidad, de modo que el último nivel es la construcción del proyecto alternativo leído a partir de la voluntad de poder.

Esos tránsitos del sujeto que dotan de sentidos comunes a la formación se pueden evidenciar en los testimonios de quienes participaron del PEPRN en calidad de estudiantes:

Como que se partía de que nosotros sabíamos de política y educación comunitaria, se partía de ese supuesto, y la mayoría de los que estábamos allí aspirábamos a saber cosas académicas, se suponía que nosotros sabíamos de política y habíamos leído, pero eso era mentira, había unos ahí que tenían cierta formación, incluso algunos años de bachillerato y otros que no, que llevábamos muchos años que no pisábamos un salón de clase y otros... completamente analfabetas, había gente que no sabía leer ni escribir entonces era complejo, ipero sí!, la gente salió aprendiendo a leer y escribir en 18 meses; hubo un muchacho que salió bien contento de ahí, yo creo que el programa fue como para perder timidez, para aprender a hablar en público, para aprender a socializar [...] había mucha gente que quería seguir, que esa vaina nos tenía que servir pa' algo. Y yo desde ahí empecé a madurar la idea de entrar a la universidad porque yo no tenía eso entre mis planes. Yo lo que sentía era ganas, yo lo que tenía era ganas. Lo que hicimos los que pudimos entrar a la universidad, fue prepararnos por nuestro lado, incluso los primeros semestres una vez dentro, yo sentía que no daba con nada, yo no entendía nada y me tuve que poner ya por mi cuenta a adelantarme. (Claudio Cruz, entrevista. En Clavijo, 2002, p. 75).

Es la voluntad del individuo lo que permite sus transformaciones, el testimonio de Claudio es claro en ello y coincide con el de otros participantes del programa:

Hoy en día yo puedo contar aproximadamente 10 compañeros que terminaron allí y hoy estudian en la UPN y sé de otros que están trabajando. Estoy seguro que de no ser así, por lo menos en mi caso, estaría en el Caguán o en otro lugar del país. (Gregorio Devia, entrevista. En Clavijo, 2002, p. 80).

La apertura del PEPRN hacia la comunidad implicó todo un reto formativo, pues de un currículo centrado y concebido para excombatientes se pasó a la propuesta de un currículo abierto y contextualizado en las realidades regionales. Así lo entiende César Zavala, otro de los fundadores de la propuesta:

[...] uno de los problemas era que con las personas que habían estado dentro del proceso, tenían el acumulado para desarrollarse y algunos de los que habían llegado no habían estado en ese proceso de combate, había un desfase y uno tenía que empezar a inventar, y algunos de los elementos que son básicos en el proyecto, se van perdiendo y en la medida en que son menos los combatientes, se va dificultando el trabajo pedagógico [...] El diseño estaba hecho para unas situaciones, cuando esas situaciones no se habían dado los productos de operación eran distintos, y era muy difícil complementar ese proceso, no es que no se pueda hacer con cualquier persona, es que las situaciones son distintas, entonces vamos a trabajar con mujeres cabezas de hogar, ellas también tienen experien- 
cias que implementar, pero el diseño es diferente al construido para un grupo de combatientes. [...] yo siento que a la comunidad hay que hacerle un trabajo más largo para que recupere la credibilidad en sí, de todos modos el que ha tenido un arma en la mano, tiene cierta dignidad entonces dice ¡si tengo algo! pero la comunidad no se siente dueña de nada, se siente pobre, ese proceso de que la gente recupere su hacer es más lento, el que un colectivo de estos empiece pensar, [sic] es un proceso histórico que acelera las cosas, entonces, la temporalidad de los 18 meses es la aceleración que depende del compromiso histórico del colectivo educativo que se esté produciendo. (César Zavala, entrevista 2002. En Clavijo, 2002, p. 90).

Involucrar a la comunidad en el proceso fue una decisión acertada, pues permitió una mayor dinámica del proyecto educativo en las regiones por cuanto fortaleció vínculos sociales del excombatiente con los pobladores de los territorios, vínculos que por dinámicas propias de la guerra se habían debilitado. La propuesta educativa siguiendo el hilo conductor de los proyectos educativos comunitarios se centró en los sentidos éticos y políticos que han constituido esos tránsitos formativos del individuo al sujeto; recordando a Sequeda (1979):

Se trata por tanto de un proceso activo y creador que se extiende a lo largo de todas las fases del desarrollo humano y que debe apuntar al desarrollo integral del adulto como miembro consciente y comprometido en la transformación histórica de la sociedad. (p. 3).

En síntesis, las propuestas de educación comunitaria encuentran en los sentidos formativos desde posturas ético-políticas, en la construcción de lo curricular e investigativo con la comunidad, atendiendo a los contextos desde una postura transformadora, aspectos comunes, que dan un lugar diferenciado a esta perspectiva educativa respecto de las tradicionales.

\section{Lo común como insurgente epistémico en la ECO}

Plantear que lo común se presenta como insurgente epistémico en la educación comunitaria, de acuerdo con las experiencias y los documentos rastreados en la Universidad Pedagógica Nacional de Colombia, implica entonces definir el elemento "insurgente" de lo común respecto a qué epistemes. Desde allí se identifican sus expresiones concretas en el campo estudiado.

De entrada, y a propósito de la educación comunitaria, es interesante retomar el origen de la palabra comunidad; Esposito (2003) nos recuerda: "No está de más señalar que communis, en su acepción primitiva, significaba no solo 'vulgar', 'popular', sino también 'impuro': sordida munera” (p. 45). Así, se puede entrar en un campo que no es hegemónico o privilegiado: el de la educación comunitaria con respecto a las licenciaturas disciplinares centradas en la obtención de títulos y la promoción regular, para revisar en esta diferenciación los sentidos de lo común en términos epistémicos.

Pensemos que lo instaurado desde las perspectivas pedagógicas y educativas, herederas de la tradición moderna, representan la cultura y el paradigma dominante, posicionado desde criterios que homogeneizan formas de producir conocimiento e incluso de hacerlo objeto de enseñanza. En esta perspectiva epistemológica que influyó en la aparición de la institución escolar moderna los ámbitos disciplinares funcionan de manera aislada, en clases separadas y con sujetos que tienen funciones claramente jerárquicas. Desde este paradigma sostenemos que "La idea de comunidad se ha utilizado, por lo general, para pensar condiciones de homogeneidad cultural y tendría como uno de sus rasgos un sistema más o menos monolítico de derechos o principios normativos" (Tapia, 2006, p. 39), que bien podrían explicar el funcionamiento de la institución escolar.

Allí no se ha dado lugar a lo común, por cuanto esta perspectiva epistemológica sustenta relaciones de dominación y discriminación, representadas en la exclusión e imposición de unos sujetos sobre otros; unos saberes sobre otros; una cultura sobre otra. Entonces en qué consiste el insurgente epistémico respecto a esta tradición pedagógica. 
Las experiencias de educación comunitaria rastreadas se ubican en una pedagogía crítica y popular, en la medida en que cuestionan los saberes instituidos, desarticulados y las "condiciones en las cuales ese saber ha sido producido" (Sequeda, 1985, p. 4). Desde esa mirada, en primer lugar encontramos que estas apuestan por un compartir los conocimientos, representado en propuestas que lograron convocar docentes y conocimientos de diferentes facultades de la Universidad, varias de ellas en busca del trabajo interdisciplinar. En torno a las prácticas comunitarias se pensó la formación de adultos, elemento central que desplazó la idea de alfabetizar al adulto en las disciplinas escolares.

La propuesta del CEA contó con el apoyo de practicantes, monitores, profesores y coordinadores de práctica de los diferentes departamentos (Educación, Matemáticas, Biología, Química, Física, Lenguas, Ciencias Sociales, etc.), a la vez que se tenía un fuerte nexo con Bienestar Universitario. De acuerdo con el profesor Mario Sequeda (1997):

[...] se tienen en un comienzo los seminarios en el CEA-UPN integrados a la práctica docente, en los cuales se trabajaban los enfoques de la práctica, los planes de estudio específicos y los procesos de interacción académico-cultural en el acontecer pedagógico cotidiano. (p. 14).

De este modo, el proceso formativo comunitario en el CEA, no pensaba en las disciplinas escolares de manera aislada, sino en cómo se encontraban para formar a los trabajadores de la Universidad, varios de ellos analfabetas.

La experiencia acumulada en el CEA incidió en la configuración de las prácticas de carácter interdisciplinario que se adelantaron en el suroriente de Bogotá, durantelos años 1984 a 1993. Estas prácticas convocaban el trabajo de los diferentes campos de conocimiento a través de articulaciones interfacultades e intersectoriales:

(...) se presentan algunos elementos relacionados con el proyecto de educación comunitaria que pretende propiciar la articulación UniversidadComunidad mediante la participación de estudiantes de las diferentes disciplinas académicas en las zonas populares, con el propósito de dinamizar los procesos de desarrollo educativo y cultural, con la participación de las comunidades para la construcción de formas de organización y autogestión solidaria que, a su vez promuevan la formación integral de los educadores de la Universidad. (Sequeda et al., 1986, p. 1).

A través de ellas se buscaban procesos de apoyo a los centros de educación básica de adultos y a algunos grupos que se reunían en el suroriente bogotano para fortalecer la relación comunidad-universidad. De esta manera, en el Preco se vinculaba la experiencia directa de los actores sociales: "En un trabajo mancomunado se realiza el diseño de los distintos campos de educación comunitaria y se construyen, desde las dinámicas participativas, las propuestas temáticas, las alternativas metodológicas y los materiales de apoyo pedagógico" (Sequeda 1997, pp. 9-10). De allí que el prolífico material didáctico elaborado en el programa, como cartillas y guías, dan cuenta de maneras distintas de abordar y cuestionar los propios contenidos escolares, a la vez que se da la invención de formas alternativas de asumirlas desde el reconocimiento del contexto, la lectura liberadora, el saber de los propios sujetos partícipes en los procesos formativos de carácter comunitario. ${ }^{2}$

En esta perspectiva también se puede ubicar el trabajo pedagógico del PEPRN, que contó igualmente con el apoyo de profesores y estudiantes de las diferentes facultades. Parafraseando a Lenkersdorf (2005), estas posibilidades de cuestionar prácticas y paradigmas sustentados en el monismo, para nuestro caso disciplinares, implican construir y recoger pluralidad de prácticas, programas, disciplinas y actores, que permiten salir del monismo para solventar de mejor manera la lucha por el reconocimiento y la transformación social. Así, en el PEPRN, este aspecto se expresa en que

Los contenidos curriculares no se debían limitar a las materias clásicas sino que debían incluir otros contenidos sobre procesos de reinserción a una vida civil, que les facilitara la vida comunitaria

2 Se rastrearon más de 30 guías y cartillas elaboradas por profesores como Dairo González y Alfonso Torres, que dan cuenta del proceso. 
urbana y les ayudara en los procesos de socialización diferentes a los de su organización original; por tal motivo a la par de las áreas obligatorias propias de la educación formal, se incluyeron otras como la gestión empresarial para apoyar la formación en la elaboración de proyectos productivos y familiarizarlos con el sector empresarial. El área de Orientación Comunitaria pretendía generar procesos de racionalización y comprensión de las reglas de juego del sistema político Nacional para facilitar su integración a las nuevas condiciones de vida. (Clavijo, 2002, p. 62).

Lo común en estas experiencias se aprecia en que el trabajo colectivo no privilegia una disciplina escolar específica; se trata de una pedagogía que responde a la construcción comunitaria del conocimiento, desde las necesidades de las comunidades y los sujetos (González y Aguilera, 2015). El acento, entonces, está puesto en conocer para transformar problemáticas propias de las poblaciones adultas excluidas, más allá del aprendizaje decodificador de la lectura y la escritura o de las operaciones matemáticas. Este reto bien invita a retomar "los saberes y prácticas antes ocultos y negados [...] para producir las condiciones materiales de reproducción de la vida social" (Gutiérrez, 2019, p. 88), es decir la reproducción de formas de vida no capitalistas.

Aquí aparece en segundo lugar como insurgente epistémico de la educación comunitaria la apuesta por desplazar los conocimientos escolares hegemónicos. Ello no indica su abandono total, sino la posibilidad de tensionarlos desde los conocimientos no oficiales, en los que las preguntas por el analfabetismo, lo comunitario y el conflicto armado permitían otras comprensiones de las realidades sociales, de los sujetos, de lo invisibilizado en los currículos oficiales.

Así, desde la década de los setenta, las propuestas de educación comunitaria representaban una posibilidad para cuestionar las prácticas educativas del momento, como las concepciones teóricas y metodológicas que orientaban el mundo académico en torno a la formación de los futuros educadores. Tal como lo señala Sequeda (1997),
No sólo se avanza en la realización de los procesos de educación básica con los trabajadores de la Universidad, sino que se viven procesos orientados hacia la recuperación crítica de las culturas populares, el reconocimiento de los contextos, la caracterización de los sujetos sociales y de las posibilidades de transformación de los centros de educación de adultos de la época. (pp. 13-14).

Por tanto, el saber popular, es decir el conocimiento propio de los sujetos y de sus condiciones de realidad, se vinculan a los procesos formativos en y para la acción transformadora (Sequeda, 1985, 1997). Esta construcción sobre lo propio desde el conocimiento reconoce las relaciones con el contexto universal, tal como lo enuncia Clavijo: "El sentido de lo propio significa la posibilidad de revalorar los saberes tradicionales frente a propuestas epistemológicas provenientes de otros contextos culturales" (2002, p. 63). En consecuencia, el pensar $y$ el conocimiento propio no se sustentan en una pretendida originalidad.

Ello indica que en la educación comunitaria lo común responde a las posibilidades de conocer con el otro, de crear alternativas teóricas, metodológicas e investigativas para comprender los contextos sociohistóricos, para abordar y producir conocimiento sobre los contenidos no oficiales inscritos en el ámbito vital y transformador de los sujetos y la sociedad,

[...] pensamos que una de las finalidades de la educación es la construcción y reconstrucción colectiva del conocimiento. Por tanto, el interrogante que guía nuestra búsqueda sería: ¿cómo investigar, educando y cómo educarnos, investigando? Este punto de vista supone que las comunidades pueden participar en la investigación y que el proceso de investigación puede ser en sí mismo educativo. Ahora bien, las investigaciones que se proponen las comunidades surgen de sus problemas y están vinculadas a acciones tendientes a resolverlos. (Torres, 1989, p. 2).

Esta perspectiva de lo común también aparece en la experiencia del Preco, con el que se buscóa través de las prácticas contribuir al mejoramiento de las condiciones de vida de las comunidades, y generar alternativas curriculares e investigativas que reconocieran las necesidades básicas de la población (Sequeda, 1997). 
De igual manera, el PEPRN permitió además de abordar la selección de contenidos oficiales y practicas evaluativas, la integración y confrontar visiones de mundo en las que se recupera el lugar del excombatiente (UPN s. f., 22). De este modo, la historia, el conocimiento del territorio, de la política, la economía y el funcionamiento de la sociedad de estos sujetos fueron reconocidos en el programa (Tellez y Guarnizo, 2017) como posibilidad formativa, que reconoce a los sujetos y sus proyectos políticos, tensionando asílas perspectivas curriculares tradicionales,

En lo concerniente a la propuesta teórico-pedagógica, el nuevo proyecto se preocupó por acceder a metodologías innovativas, fue entonces cuando nuevamente se comenzó a hablar de dialogo de saberes - encuentro de saberes- para intentar interrelacionar experiencia con conocimiento científico, se incorporaron además métodos como la crítica de imágenes y el análisis de conflicto, esto para construir junto con el estudiante nuevos sentidos e interpretaciones. (Clavijo, 2002, p. 64).

Esta comprensión de lo común como insurgente epistémico se expresa al revertir relaciones pedagógicas tradicionales, basadas en "enseñar, transmitir e incluso aprender, pues su sustento está en el compartir, noción que de suyo asume que todos los sujetos son sujetos de conocimiento" (González y Aguilera, 2015, p. 5).

En tercer lugar, sostenemos que lo común en la educación comunitaria se expresa en oposición al ideal comeniano "enseñar todo a todos" y de la misma manera, ya que al subvertir la relación de los saberes escolares en los que se abordan contenidos que desbordan las disciplinas escolares, en efecto no se enseña ese "todo" predeterminado. El sentido de lo común en esta perspectiva educativa no se recoge en el ideal pansófico, pues el enseñar está referenciado directamente a la didáctica de las disciplinas escolares. En el ámbito comunitario podemos pensar lo contrario, allí el proceso formativo está mediado por la corporeidad humana que incluye el pensar, el sentir, el imaginar, el razonar y el actuar (Lenkersdorf, 2005). El pensar y la producción de conocimiento en la educación comunitaria acuden a la invención, la creación y a los lenguajes no racionales.

Pero tampoco se enseña a todos, niños y jóvenes, pues en este campo de la educación comunitaria el ingreso del adulto analfabeta, de los adultos no escolarizados, excombatientes, líderes o dinamizadores sociales cuestionan ese a todos definido por la edad de ingreso y permanencia en la institución escolar. Aquí, ese todos excluye, como ha excluido, a sujetos por su condición étnica, etaria, de género, de discapacidad, de identidad sexual y por su condición social.

El insurgente epistémico común de la educación comunitaria lo detona el reconocimiento de la diferencia, eso que pone en crisis los sistemas homogéneos instituidos con la pedagogía moderna. Ese a todos en definitiva, históricamente, no nos ha cobijado a otros. Lo vemos en el CEA, donde "El Centro de Educación de Adultos como creación colectiva de los trabajadores, estudiantes, profesores y directivos universitarios permite comprender el importante acicate que en los setentas significaba la superación cultural de los actores universitarios" (Sequeda, 1997, p. 13). Así, el Centro abrió las puertas a empleados de la cafetería, aseadoras, conductores, celadores, jardineros, funcionarios de la biblioteca y operarios telefónicos, entre otros.

Allí se reconocía a aquellos sujetos que por diferentes razones no hicieron parte del mundo escolar a temprana edad. Pero no bastaba con incluirlos en procesos formativos que les posibilitaran alfabetizarse; se trataba de participar en la construcción de alternativas de vida. Pasar de firmar con la huella dactilar a la escritura de un nuevo mundo fue la posibilidad de varios de estos trabajadores para cambiar sus condiciones de existencia, acompañados de una mirada crítica del mundo.

En la experiencia del Preco, los sujetos del suroriente de Bogotá se identificaban como parte de los sectores populares,

[...] habitantes del suroriente que garantizan su subsistencia dentro de la versatilidad muy propia de los sectores populares, la cual toma forma en la llamada "economía del rebusque". Los que han 
logrado incorporarse a la fuerza de trabajo asalariado de la ciudad se desempeñan básicamente en el sector de servicios y de construcción; los que no han podido hacerlo, se desempeñan como trabajadores independientes que, por contrato y en forma intermitente, ofrece sus conocimientos en diversas artes y oficios para los diferentes sectores económicos. Finalmente, una minoría ha logrado generar algún tipo de micro-empresa e inclusive contribuir a crear nuevas formas de empleo para otras personas de la comunidad (Sequeda et al., 1985, pp. 4-5).

En el convocar de manera diferencial a los sujetos de la educación comunitaria encontramos que lo común es lo que nos hace diferentes. Desde esta perspectiva, lo común no excluye lo diverso pero tampoco lo junta en lo igual. Al respecto, en la experiencia del PEPRN, los sujetos excombatientes, además de su condición de actores dentro del conflicto armado, eran reconocidos de acuerdo a su condición étnica. Por ejemplo,

[...] desde las experiencias de algunos participantes del programa, el enfoque diferencial que se utilizó fue un aporte fundamental. Como ejemplo de ello se encuentra lo sucedido en el proceso educativo con el MAQL en el cual se creó un modelo de educación étnica, apoyado con el Consejo Regional Indígena del Cauca (CRIC), el Movimiento Indígena y algunas universidades, esto nos muestra que el programa se preocupó por comprender y reconocer las particularidades de cada insurgencia y por generar formas acertadas de abordar el tema educativo. (Téllez y Guarnizo, 2017, p. 60).

También respondió a otros sujetos excluidos del monopolio de la escuela tradicional, ya que

[...] este programa en una tercera fase se reglamentó en la Resolución 3000 de 1996, acogiendo población divergente como lo serían trabajadoras sexuales, también población vulnerable como madres comunitarias, jóvenes de escasos recursos y empleados oficiales de la Registraduría, culminando su accionar este programa en 1996, otorgando para entonces 675 títulos de bachiller a desmovilizados y 1200 al resto de la comunidad. (Téllez y Guarnizo, 2017, p. 44).
El reconocimiento de las diferencias se expresaba en el PEPRN en la valoración de las historias dispares y en los diferentes niveles formativos que tenían los excombatientes (Rojas, citado en Clavijo, 2002). Ello con la intención de formar un hombre distinto para un modelo social distinto.

En síntesis, consideramos que lo común como insurgente epistémico en la educación comunitaria atiende a procesos que no jerarquizan los conocimientos (ciencias, saberes, epistemes), los sujetos (educadores, adultos, pobladores, militantes etc.), la cultura (popular, letrada), lo político (poder, Gobierno, instituciones). Ello indica que lo común no jerarquiza ni privilegia, requiere de lo colectivo, como posibilidad para cuestionar los monismos metodológicos, pedagógicos, investigativos, políticos, culturales, e incluso como medio para vincular críticamente la misma cultura dominante.

Lo común como posibilidad colectiva crítica implica el reto de seguir pensando reinvenciones de estos sentidos de lo común, que coadyuven a la construcción de

[...] nuevas instituciones y espacios políticos, sociales y económicos que nos permitan configurar estructuras de organización, formas y procesos de deliberación y dirección, compuestos por la presencia igualitaria de todos los pueblos y sus formas sociales y políticas [...]. (Tapia, 2006, p. 6).

Una alternativa a la configuración de estos nuevos espacios podría estar en pensar cómo articular a nuestros contextos algunas formas de poder que circulan en los saberes ancestrales. En palabras de Gladys Tzul (2015):

Estas formas de gobierno comunal indígena producen y controlan los medios concretos para la reproducción de la vida cotidiana mediante, por lo menos, tres formas políticas, a decir: el k’ax kol (o trabajo comunal) que da vida a los medios concretos para la vida; las tramas de parentesco, poderosa y a la vez contradictoria estrategia, que se usa para defender la propiedad comunal del territorio y para organizar el uso del mismo; y la asamblea como forma comunal de deliberación para resolver problemas cotidianos [...] (pp. 128-129). 
De estas tres formas de gobierno indígena, dos se han visto reflejadas en los contextos de las experiencias en cuestión: el trabajo comunal y la asamblea deliberativa, allí la participación colectiva en la construcción de lo que nos es común permitió rediseñar desde la educación comunitaria formas de vida capaces de impugnar el orden económico, cultural y político impuesto, toda vez que marcaron diferencias con lo formalmente institucionalizado. Específicamente, el CEA y Preco fueron testimonios fehacientes, cuando aún en condiciones adversas lograron dar cumplimiento a gran parte de sus horizontes, en ello la solidaridad, la participación y el compromiso fueron principios fundantes para que estas propuestas pudieran sostenerse.

\section{Solidarios en un trabajo creativo creador}

Consideramos que un profundo sentido de lo común en la educación comunitaria encuentra un correlato fundamental en la solidaridad, como principio de pensamiento y acción que orienta el trabajo educativo con los sujetos excluidos del sistema social. Encontramos que al hablar de "Solidarios en un trabajo educativo creador", lema que aparece en títulos y textos del profesor Mario Sequeda Osorio a propósito de lo que fue el Centro de Educación de Adultos en los setenta, remite a la construcción colectiva "desde la óptica de los actores mismos como un trabajo educativo solidario, crítico y creador" (Sequeda, 1985, p. 13). Solidaria es la potencia que se crea ante el fatalismo material y subjetivo que se impone desdes el modelo económico imperante, de allí que se considere un principio que "Impugna el orden económico y político vigente" (Torres, 1997, p. 12). Las posibilidades que desde la educación comunitaria han ido generando otras perspectivas de mundo posible requieren, parafraseando a Raquel Gutiérrez (2019), de un proceso complementario de disolución-creación. Si bien no encontramos en el rastreo histórico realizado experiencias y propuestas que expresen la disolución de la escuela capitalista, en ellas se mantiene vigente la apuesta por crear otras perspectivas de vida.
Lo común-solidario en términos de lo político aparece en los procesos que colectivizan decisiones, prácticas, relaciones y proyectos comunes. En las experiencias educativas abordadas se reconoce la vivencia de lazos de fraternidad, de afecto y respeto mutuo, que permitieron confrontar las relaciones escolares tradicionales, para generar prácticas políticas en las que principios como la horizontalidad y la desconcentración del poder van cobrando vida en el ámbito educativo comunitario. Así, por ejemplo, las jornadas evaluativas del CEA constatan que

Estas Jornadas van permitiendo decantar un conjunto de principios y objetivos, una estructura curricular específica y definir, por consenso, un reglamento interno que permite una acción educativa organizada en la cual se transparenten los valores de la criticidad, la creatividad, la autonomía y la participación solidaria en función del beneficio colectivo. (Sequeda, 1980, p. 6).

Es en el trabajo solidario y en la colectivización de las decisiones que lo común cobra vida, por cuanto vincula las tensiones, los acuerdos y disensos para permitir pensar en el beneficio colectivo. Por tanto, una propuesta como la construcción de un currículo comunitario "está íntimamente ligado a la concepción misma de educación comunitaria como proceso social de dinamización del potencial creador de las personas en comunidad, a partir de la problematización y concientización de sus condiciones de existencia para con su participación organizada, transformar la realidad" (Sequeda et al., 1986, p. 176). En esta misma línea, el Preco trabajaba colectivamente en la "definición de objetivos compartidos, y las acciones que es preciso adelantar demandan, en la autogestión, asumir tareas por parte de los distintos integrantes de los grupos comunitarios" (Sequeda et al. 1985, p. 3). Al respecto, resulta sugerente que el asumir tareas por parte de cada integrante del mundo se encuentra con un sentido de lo común que aparece en la política del nosotros tojolabal (Lenkersdorf, 2005), en la que se exige el aporte de cada sujeto para contribuir a la vida comunitaria. $\mathrm{O}$, como lo menciona Tapias (2006), estas prácticas se asocian a la construcción 
de formas de gobierno común que indican que cada uno debe asumir estas tareas o roles decisivos por lo menos una vez en la vida. Este aspecto se hace evidente en la asunción de procesos de investigación colectivos, de diseños metodológicos participativos y de la definición de objetivos comunes.

De esta manera, la colectivización de las decisiones en las experiencias educativas comunitarias permite recrear otras formas de tomas decisiones, $\mathrm{o}$, como diría Lenkersdorf (2005), se recrea un nosotros comunitario, que se realiza en la toma de decisiones colectivas como proceso que permite pensar el actuar por fuera del Estado - o de lo instituido-, a la vez que reformarlo en términos de establecer otras formas de gobierno,

Lo participativo no es solamente una dimensión técnica del proceso educativo sino una dimensión esencial, por cuanto lo que queremos es un individuo participante, transformador y a su vez queremos que ese sujeto se forme a través de una metodología que le permita ejercitar su capacidad de decisión, es decir su libertad. (Sequeda, 1985, p. 5).

Allí, encontramos rasgos distintivos para provocar y andar una política de lo común en el ámbito educativo-comunitario, que tal como la define Gutiérrez (2017), bebe de las prácticas políticas de los pueblos indígenas latinoamericanos y se expresa concretamente en la oposición y creación antagónica "al despojo o apropiación privada de lo entre todos producido, incluyendo la privatización de la capacidad de decisión que se amalgama en las modernas formas liberales de lo político y la política" (Gutiérrez, 2017, p. 107). Por tanto, la experiencia de lo común desde la educación comunitaria genera prácticas políticas en las que no se concentran las decisiones; en su lugar, se implican formas colegiadas, asamblearias o de cogobierno para definir el destino común del proceso formativo y sus sentidos transformadores.

Otro rasgo que aparece en torno a los sentidos de lo común en las experiencias de educación comunitaria atiende a la búsqueda y/o reivindicación de formas de vida capaces de sobrevivir al capitalismo. En esta perspectiva, la búsqueda de lo común en lo comunitario puede entenderse como forma de creación ante lo ausente:

Por lo tanto, communitas es el conjunto de personas a las que une, no una "propiedad", sino justamente un deber o una deuda. Conjunto de personas unidas no por un "más" sino por un "menos" una falta, un límite que se configura como un gravamen, o incluso una modalidad carencial, para quien está "afectado", a diferencia de aquel que está "exento" o "eximido". (Esposito, 2003, pp. 29-30).

Es decir que en los procesos de educación comunitaria se reconocen las deudas educativas, políticas, económicas y culturales, desde lecturas críticas que permiten crear, con el vínculo solidario y del reconocimiento, el andar formas de sociabilidad y de prácticas políticas y económicas de carácter emancipatorio (Torres, 1997).

Se trata de pensar lo común como invención, no como criterio, como generador del vacío reconfortante que puede materializarse de diversas maneras en la economía solidaria, la autonomía alimentaria, la autogestión, o como lo profundiza Gutiérrez $(2017,2019)$ la reproducción social de la vida. Para las experiencias revisadas se trataba de "dinamizar los procesos de apropiación, expresión y recreación de la cultura popular, a clarificar la participación de las comunidades en la vida productiva y a propiciar la organización y autogestión solidaria en torno a sus necesidades básicas" (Sequeda et al. 1986, p. 5). Ese horizonte educativo posiciona de entrada la posibilidad de recrear formas de vida y existencia que dignifiquen la condición humana, por ello no se inscriben en los procesos educativos que exaltan el camino al desarrollo capitalista.

Todo este recorrido, que a la vez que contribuye a hacer visible uno de los saberes negados y excluidos de las tradiciones pedagógicas en nuestra universidad, dibuja retos inconmensurables para seguir imaginando y aportando propuestas educativas comunitarias que vienen recuperando otras formas de vida posibles y que se oponen, tensionan o se crean por fuera del mundo capitalista. Esta tarea se inscribe en la pregunta que lanzan varios pueblos 
indígenas, campesinos y urbano-populares, y es ¿qué educación, para qué sociedad? Si seguimos recuperando las perspectivas que desde la construcción de lo común ha logrado sobrevivir y confrontar la producción del sistema capitalista, tendremos que indagar cómo en estos procesos se sostienen propuestas formativas y educativas que están dibujando la producción de formas de vida comunitarias.

\section{Referencias}

Aguilera Morales, A., González Terreros, M. I., Torres Carrillo, A. (2015). Reinventando la comunidad y la política desde las organizaciones locales. Formación de subjetividades, sentidos de comunidad y alternativas politicas en procesos organizativos locales. Bogotá: UPN.

Clavijo Ramírez, A. (2002). Programa Educativo para la Paz y la Reconciliación Nacional: de una propuesta alternativa a una práctica funcional innovativa (tesis de maestría). Universidad Pedagógica Nacional, Bogotá, Colombia.

Clavijo Ramírez, A. (2016). Educación popular y educación comunitaria: ¿̇esignificación o refundamentación? En W. J. Díaz Ramírez, Y. P. Sanabria Téllez (comp.-coautores). Pedagogías críticas y emancipatorias: un homenaje a Paulo Freire (pp. 147-160.) Bogotá: Universidad Distrital Francisco José de Caldas.

Clavijo Ramírez, A. (2017). La educación comunitaria en el contexto de las propuestas de práctica: reflexiones desde la experiencia con educadores en formación. En Polifonías de la educación Comunitaria y Popular (pp. 31-45). Bogotá: UPN.

Comenio, J. A. (1988). Didáctica magna. México: Editorial Porrúa.

Esposito, R. (2003). Nada en común. En Communitas. Origen y destino de la comunidad (pp. 21-48). Buenos Aires: Amorrortu.

Gadotti, M. (1993). La educación comunitaria y los movimientos populares. En F. Gutiérrez (comp.). Educación comunitaria y economía popular. Costa Rica: PEC-Heredia.

González, M. I., Aguilera Morales, A. (2015). Una Universidad "de" y "para" el movimiento indígena. Revista Cisma, 6, 1-18.
Gutiérrez Aguilar, R. (2017). Más allá de la "capacidad de veto": el difícil camino de la producción y la reproducción de lo común. En Horizontes comunitario-populares. Producción de lo común más allá de las políticas estado-céntricas. Madrid: Traficantes de Sueños.

Gutiérrez Aguilar, R. (2019). Común, ¿hacia dónde? Metáforas para imaginar la vida colectiva más allá de la amalgama patriarcado-capitalismo y dominio colonial. Producir lo común. Entramados sociales y luchas por la vida. El Aplantle. Revista de Estudios Comunitarios.

Lenkersdorf, C. (2005). Filosofar en Clave Tojolabal. México: Miguel Ángel Porrúa.

Quintana, J. M. (1991). Pedagogía comunitaria. Perspectivas mundiales en educación de adultos. Madrid: Narcea.

Sequeda Osorio, M. (1998). Educación comunitaria, cultura democrática, proceso de paz: construyendo amaneceres. En Documentos de trabajo. Maestría en Educación Comunitaria. Bogotá: Universidad Pedagógica Nacional.

Sierra, P. (2000). Algunas reflexiones iníciales sobre la educación popular y su relación con la educación comunitaria. En Relaciones entre la educación popular y Educación Comunitaria. Bogotá: Documentos de trabajo. Maestría en Educación Comunitaria. Universidad Pedagógica Nacional.

Tapia, L. (2006). La invención del núcleo común. Ciudadanía y gobierno multisocietal. Bolivia: Ed. Muela del Diablo.

Téllez, M. y Guarnizo, A. (2017). La educación en procesos de posacuerdo: una mirada a los escenarios educativos para excombatientes en Colombia (trabajo de pregrado), Bogotá: Universidad Pedagógica Nacional, Colombia

Torres Carrillo, A. (1997). Modernidad y nuevos sentidos de lo comunitario. Pedagogía y Saberes 10, 5-14.

Torres Carrillo, A. (2002). Reconstruyendo el vínculo social. Lo comunitario en tiempos globalizados. Administración y Desarrollo, 37, 24-45.

Torres Carrillo, A. (2013). El retorno a la comunidad. Bogotá: Cinde-El Búho.

Tzul Tzul, G. (2015). Sistemas de gobierno comunal indígena: la organización de la reproducción de la vida. $E l$ Aplantle, 1, 125-139. https://horizontescomunitarios. files.wordpress.com/2017/01/elapantle.pdf. 
Zabala, V. (1990). Principios y elementos básicos del Programa Experimental Piloto Para la Paz y la Reconciliación Nacional. Bogotá: Corporación Tres.

Zemelman Merino, H. (1992). Educación como construcción de sujetos sociales. La Piragua 5, 12-18. Santiago de Chile: Ceaal.

\section{Referencias primarias}

\section{Archivo Personal Mario Sequeda Osorio}

Sequeda Osorio, M. (1977). Solidarios en un esfuerzo educativo y creador. Universidad Pedagógica Nacional.

Sequeda Osorio, M. (1979). Alternativas para la formación y capacitación de educadores de adultos. Ponencia presentada en la XIII Reunión Nacional de Coordinadores de Educación de Adultos Centro de Educación de Adultos. Departamento de Educación, Universidad Pedagógica Nacional.

Sequeda Osorio, M. (1980). El Centro de Educación de Adultos-upN. Un proyecto de educación popular. Balance y perspectivas. Bogotá: UPN.

Sequeda Osorio, M. (1985). A propósito del Movimiento Pedagógico. Educación y comunidad hacia una alternativa pedagógica. UPN: inédito.

Sequeda Osorio, M. (coord.), Franc, M., Posada, J., Mantilla, A., Torres de Vergar, M. y Aponte, G. (1985). La formación en la acción del educador comunitario de carácter polivalente. Elementos para una propuesta. En Seminario Técnico regional sobre Formación de Educadores Polivalentes para comunidades rurales en el marco del proyecto principal de educación para America Latina y el Caribe. UPN-Departamento Administrativo de Bienestar Social. Centro Comunitario La Victoria. Paipa.

Sequeda Osorio, M. (1997). Hacer memoria, abrir caminos, sembrar esperanzas desde la óptica de la educación comunitaria, la cultura democrática y la biopaideia jovial. UPN. Inédito.

Sequeda Osorio, M. (s. f. a). Relacion universidad-comunidad. Hojas sueltas.

Sequeda Osorio, M. (s. f. b). El CEA-UPN: evaluación crítica. S. d.

\section{Archivo Central Universidad Pedagógica Nacional}

Universidad Pedagógica Nacional (s. f.). Programa Educativo Para la Paz y la Reconciliación Nacional. Proyecto Técnico y Económico para el desarrollo del Programa en Córdoba, Uraba, Norte de Santander, Santander, Caquetá, Bolívar, Antioquia, Cauca, Santa Fe de Bogotá, Cali y Medellín.

\section{Archivo personal Alfonso Torres Carrillo}

Torres Carrillo, A. (1989). La Enseñanza de las Ciencias Sociales con Adultos y Jóvenes de Sectores Populares Urbanos (Práctica en Educación Comunitaria). Universidad Pedagógica Nacional, Facultad de Artes y Humanidades, Departamento de Ciencias Sociales. Bogotá: UPN.

\section{Archivo Personal Amadeo Clavijo Ramírez}

Gallo Arbeláez, M. E. (1994). Programa Educativo Para la Paz y la Reconciliación Nacional: Educación de Jóvenes y Adultos Para la Ciudadanía Plena. Bogotá: Universidad Pedagógica Nacional. 